\title{
Is Cork a Good Closure for Virgin Olive Oil?
}

\author{
Ofélia Anjos ${ }^{1,2} a^{*}$, Luís Coutinho ${ }^{1, b}$, Cecília Gouveia ${ }^{1, c}$ and Fátima Peres ${ }^{1,3, d}$ \\ ${ }^{1}$ Instituto Politécnico de Castelo Branco, Castelo Branco, Portugal \\ ${ }^{2}$ Centro de Estudos Florestais, Instituto Superior de Agronomia, Universidade de Lisboa, \\ Lisboa, Portugal. \\ ${ }^{3}$ LEAF, Instituto Superior de Agronomia, Universidade de Lisboa, Lisboa, Portugal. \\ a*ofelia@ipcb.pt, ${ }^{b}$ tojeiraprodutosbiologicos@gmail.com, ${ }^{c}$ cgouveia@ipcb.pt, ${ }^{d}$ fperes@ipcb.pt \\ Keywords: Cork Stopper; Screw Cap; Storage; Virgin Olive Oil
}

\begin{abstract}
Most of the studies on olive oil preservation during storage are focused on the type of conditioning rather than on the most efficient type of bottle seal to be used. However, the bottle closure is also an important issue because of the negative impact that oxygen has on olive oil quality and flavor.

The aim of this study was to assess the performance of a natural cork stopper as a closing system of glass bottles for olive oil.

To evaluate the effect of a bottle closure, a storage trial over 24 months, after harvest, was performed comparing the effect of three types of glass bottle closure on virgin olive oil quality: screw cap, natural cork and bar top cork stopper. The bottle neck of those with a natural cork stopper was covered with bee's wax. The results for quality criteria showed that all three types of bottle closure acted in a very similar way for most of the studied parameters. However, based on FTIR-ATR spectral information it was possible to separate the samples with different closure systems at the end of the storage period. These differences could be given by the retention of some volatile compounds detected by sensory evaluation.

The olive oil oxidation parameters were not highly affected by the cork stopper. However, for olive oil quality characterization with a cork stopper, more studies are needed for the volatile composition.
\end{abstract}

